\title{
The Fabrication of Microretarder for In-cell Stereoscopic LCD using Reactive Liquid Crystal
}

\author{
Gwo-Feng Tseng ${ }^{2}$,Chao-Hsu Tsai ${ }^{1}$, Hoang Yan Lin ${ }^{2}$, Wan-Jian Huang ${ }^{1}$, \\ Kuo-Chung Huang ${ }^{1}$, Kuen Lee ${ }^{1}$ \\ ITRI/EOL,195,Sec.4, Chung Hsing Rd. Chutung, Hsinchu 310 Taiwan, R.O.C. ${ }^{1}$ \\ National Taiwan University, Institute of Electro-optical Engineering ${ }^{2}$
}

\begin{abstract}
A microretarder for stereoscopic display is a film consisting of horizontal or vertical stripes with alternating null and half-wave phase retardation states. An LCD with a microretarder attached on the front side can display good-quality stereoscopic images when viewed with polarization glasses. It is now the easiest and cheapest way to present stereoscopic images on a flat-panel display. However, the space caused by the glass between the retarder layer and the LC layer of the panel seriously limits the vertical view angle, which, in turn, limits the application of this technology. In this paper, a process for thin-film microretarder is developed using reactive liquid crystal. The material and the process are essentially compatible with current LC panel processes. Since the thin-film microretarder is to be fabricated in the cell of an LC panel, the retarder layer and the LC layer can be fabricated as close to each other as possible. A nearly unlimited $3 \mathrm{D}$ view angle can be achieved for the display.
\end{abstract}

\section{Key words: stereoscopic display, 3D, microretarder, reactive liquid crystal}

\section{INTRODUCTION}

By the progress on display technologies in recent years, 3-D displays have been built in many different ways which can be classified into two major types. One is non-glass (autostereoscopic) display including holographic display, volumetric display, parallax barrier, and micro-lenticular display which still suffer from some difficulties like view angle, image quality, data transfer rate, etc. The other way that we call stereoscopic display is to reproduce 3-D images by using shutter glasses or polarized glasses to separate the parallax images, which can provide us the higher quality images . In this paper, on the basis of nowadays LCD technology, we provide a microretarder layer inserted into the interior LCD cell instead of building it on the exterior layer. Comparing with the traditional method, which attaches microretarder film to the exterior LCD glass, the in-cell method shrinks the thickness of the proposed structure, expands viewing angle, improves optical alignment progress, and provides a better protection to the microretarder layer. Here we use reactive liquid crystal material (RMS03-001C) to manufacture microretarder thin film by using spin coating method.

\section{PRINCIPLE AND THE STRUCTURE}

Using binocular cues to reproduce 3-D images for the end user is the major method in nowadays 3-D display system. Binocular vision provides humans with the depth perception derived from the slight differences in the view-angle of homologous or corresponding images. Therefore, construction for building binocular vision is the key-point for 3-D display.

In this paper, a polarization multiplexed system using microretarder film has been demonstrated. A microretarder is a film consisting of 0 and half-wave optical retardation states on alternate horizontal or vertical stripes. For half-wave retardation state, it provides $\pi$ phase retardation by material birefringence:

$$
\delta=k d n_{x}-k d n_{y}=k d \Delta n=\pi
$$

When passing through $\pi$ phase retardation, the polarization state of incident light rotates a $2 \theta$ angleas shown in Fig. 1,

Stereoscopic Displays and Virtual Reality Systems XIV, edited by Andrew J. Woods, Neil A. Dodgson,

John O. Merritt, Mark T. Bolas, lan E. McDowall, Proc. of SPIE-IS\&T Electronic Imaging,

SPIE Vol. 6490, 64900W, @ 2007 SPIE-IS\&T · 0277-786X/07/\$18 
where $\theta$ is the angle between the polarization direction of the incident light and the optical axis of the retardation film.

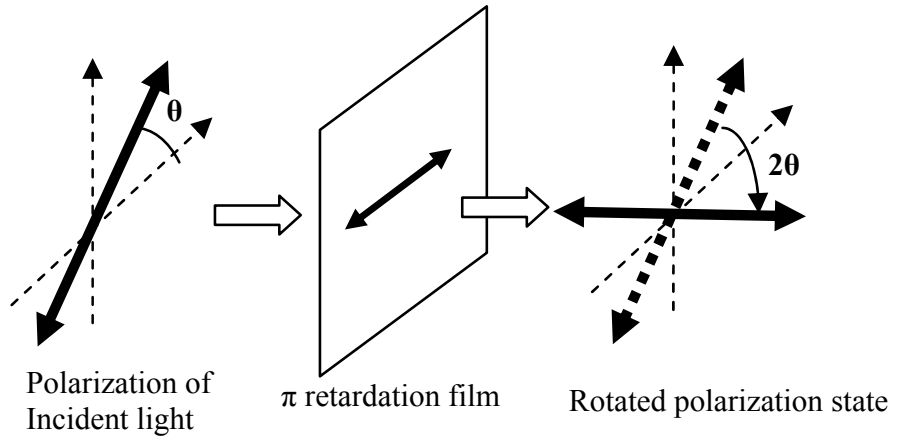

Fig. 1. The polarization state transformed by the $\pi$ retardation film, is mirrored with respect to the optical axis ( $\mathrm{x}$ direction in this case) by an angle $\theta$.

With the orthogonal polarization states of light after passing through the alternate 0 and $\pi$ retardation areas, a binocular images display system can be built with left-eye and right-eye images interleaved horizontally. In Fig. 2., a microretarder film with horizontal stripes of alternated 0 and $\pi$ retardation is aligned and attached to a polarized display panel like LCD.The polarization of the light passing through the $\pi$ retardation area will rotate 90 degrees, while that of the light passing through the 0 retardaion area maintain its polarization state. When the horizontally interleaved left-eye and right-eye images are displayed on the polarized display panel, the user, wearing orthogonal polarization glasses, will be able to view the 3-D images. .

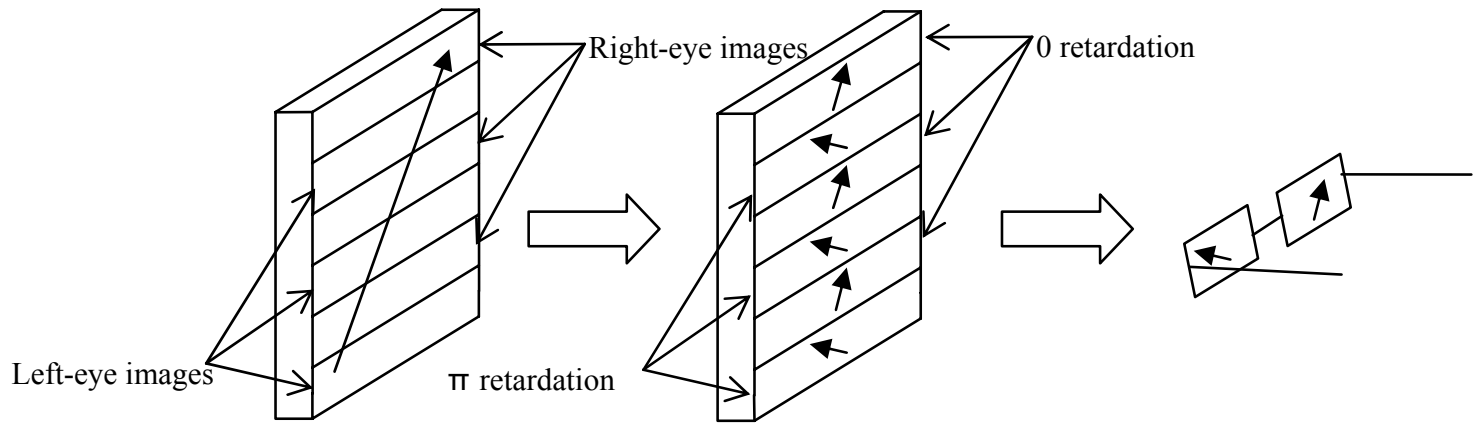

Fig. 2. Operation of the demonstrated binocular images system with polarized glasses

A stereoscopic display system which used LCD panel attached by a microretarder in the front side has been demonstrated [1]. However, low-range viewing angle suffered by the thickness of LCD front-side glass violently restricts the image quality. An "in-cell structure" is demonstrated in this paper. In Fig. 3., it shows the proposed structure that combined with (from left to right side) backlight module, polarizer, ITO layer, LC layer, ITO layer, color filter, analyzer, microretarder layer, and glass. The viewer at right side in the end is wearing polarized glasses which have orthogonal polarizer filters to get the binocular images. In the in-cell structure, the biggest difference from the conventional architecture is inserting the specially fabricated microretarder to the inner side of the glass instead of attaching it on the outer side of the front glass. In this case, the distance between the microretarder and the LC layer is reduced from around $1 \mathrm{~mm}$ to a few microns, and, in turn, the vertical view angle is significantly enhanced. 


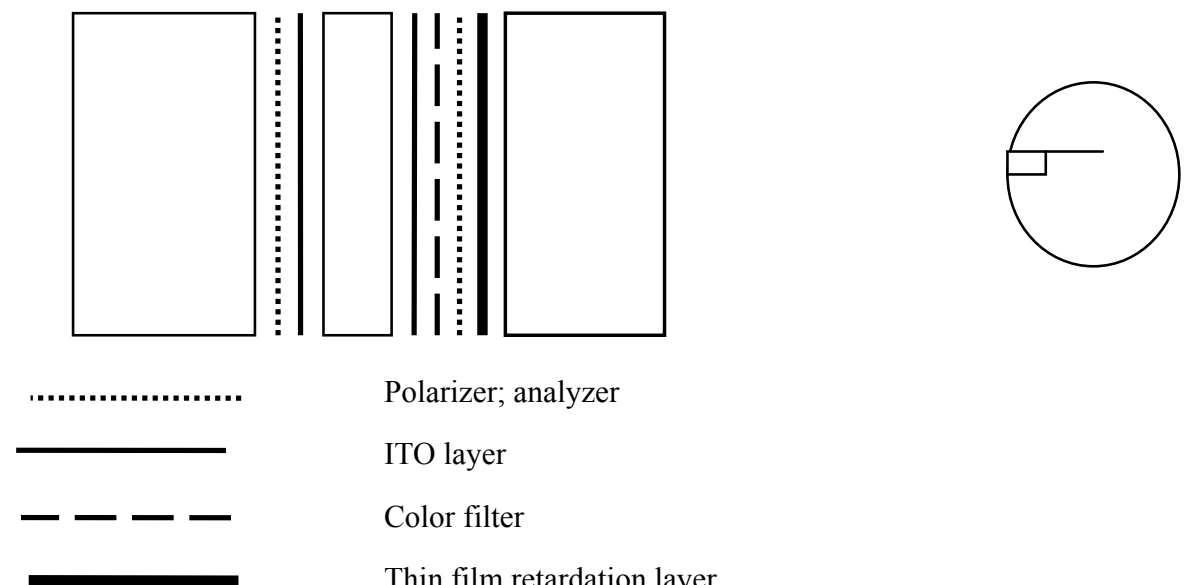

Fig. 3. The proposed structure of in-cell microretarder.

\section{THERMAL AND UV PROCESS}

There had been successful methods in fabricating microretarder, including laser scanning method[1] and chemical etching method. Nevertheless, the currently used material of the film makes it impossible to insert the microretrder film into the inner side of the LCD glass, because polymeric retardation film can not survive from the rigorous LCD process. In this paper, we use liquid crystal based material to fabricate the microretardation film and will focus on the experiment of the forming of the retardation film and the patterning.

Before patterning the retardation film, the fundamental properties of the reactive liquid crystal material RMS-03-001C in forming retardation film were studied in detail. The RMS-03-001C is in liquid crystal state at room temperature and will transit from liquid crystal to isotropic state during $70^{\circ} \mathrm{C}$ to $100^{\circ} \mathrm{C}$, and become isotropic state when the temperature arise to $100^{\circ} \mathrm{C}$ and above. The relationship of the retardation v.s. temperature is shown in Fig.4. Another factor which can be used to control the retardation value is the thickness of the reactive liquid crystal film, which is related to the parameter of the coating process, e.g. the spin speed. The relationship of the retardation v.s. spin speed is shown in Fig. 5.

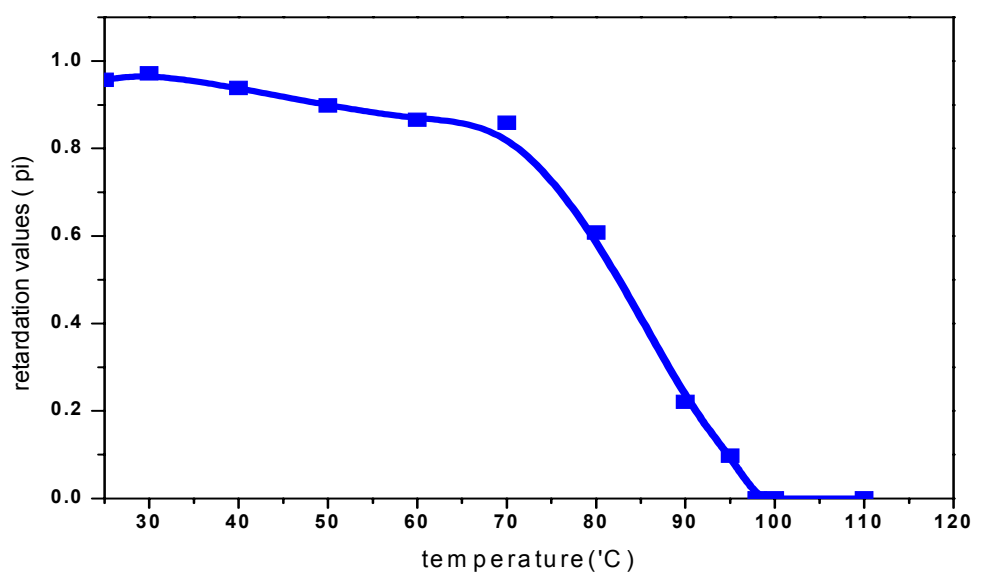

Fig. 4. The relation between temperatures and retardation values. 


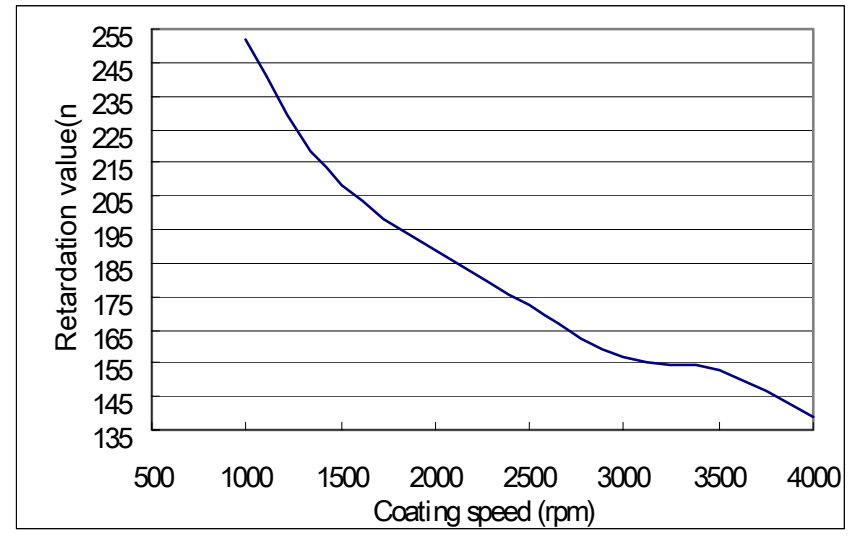

Fig. 5. The relation between spin speeds and retardation values.

To fabricate the stripes pattern on the retarder film using the reactive liquid crystal material, we chose the thermal and UV process. First, an alignment layer is coated on glass substrate. Rubbing process was taken to form the alignment structure on it. reactive liquid crystal film was then spread on top of the alignment layer by a spin coater. The retardation effect can easily be seen at this step. However, the retardation value of the film now is different from that after all processes because of the difference of the thickness. The glass substrate with reactive liquid crystal film was then put on an oven of $80{ }^{\circ} \mathrm{C}$ for 30 seconds, allowing the solvent to escape. Then the substrate was moved to an temperature controllable and UV equipped oven filled with nitrogen. The purpose of the nitrogen is to prevent the interaction of oxygen and the reactive liquid crystal layer. The mask with required pattern was then put on top of the reactive liquid crystal film. When the oven is at room temperature, turn on the UV light source. The reactive liquid crystal layer under the transparent area of the mask was cured and fixed at the state with retardation. Then remove the mask of the pattern and rise the oven temperature to $100^{\circ} \mathrm{C}$. The fixed area of reactive liquid crystal layer remained the state with retardation, while the area which originally under the opaque area of the mask become isotropic The variation of the retardation in each step is shown in Fig. 6. Turn on the UV light source again. The whole area of the film was fixed. A microretarder with two states of retardation on a prescribed pattern was formed. The procedures are summarized as Fig.7.

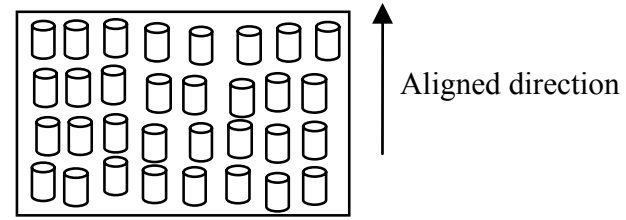

(a)

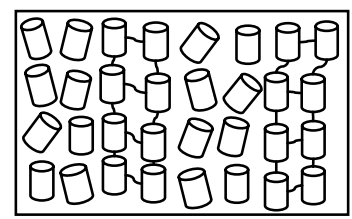

At 100

(c)
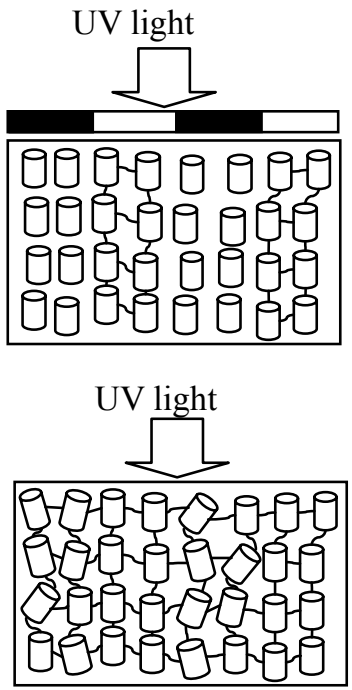

(b)

At 100

(d)

Fig. 6. Detail alignments of liquid crystal molecules for each fabrication step: (a) coating with aligned direction (b) UV exposing at room temperature through the mask (c) heating substrate at $100^{\circ} \mathrm{C}$ (d) $\mathrm{UV}$ exposing at $100^{\circ} \mathrm{C}$ again 


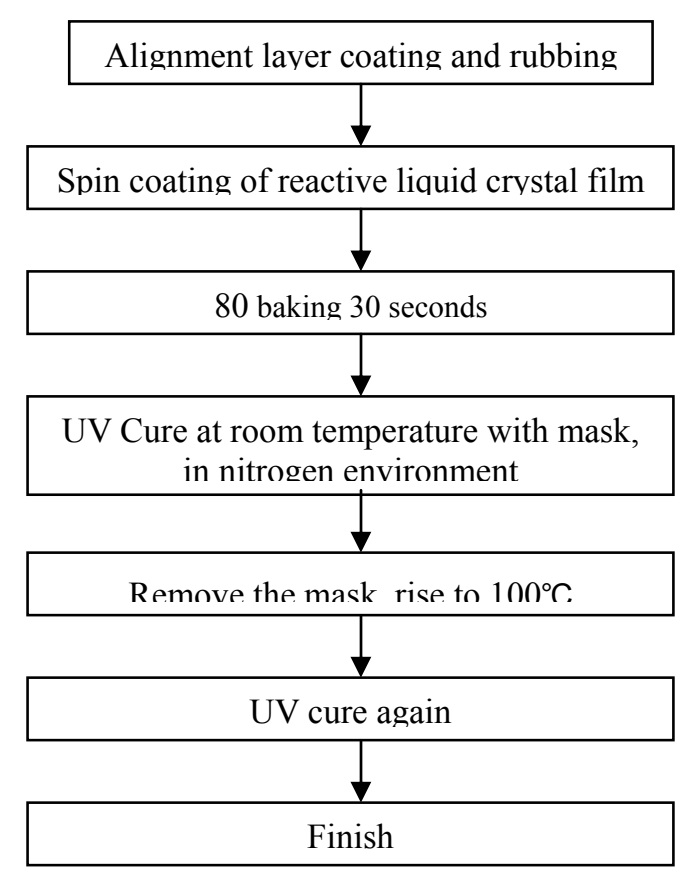

Fig. 7. The procedures of microretarder fabrication using reactive liquid crystal material

\section{EXPERIMENTS AND MEASUREMENT}

The above procedures were used to fabricate the microretarder with stripes pattern. However, a problem happened and seriously limited the minimum line width achievable by the procedure. It's because the mask has to be attached to the reactive liquid crystal layer before it is cured. In that case, the film of the reactive liquid crystal layer is in it's liquid state. Even after UV curing, part of the film, the area which is under the opaque area of the mask, is still in liquid state. Attach of the mask to a liquid film and remove will seriously destroy the uniformity of the film. To avoid destruction of the film, we keep a very small gap between the mask and the film. A microretarder made by this method is shown in Fig. 8

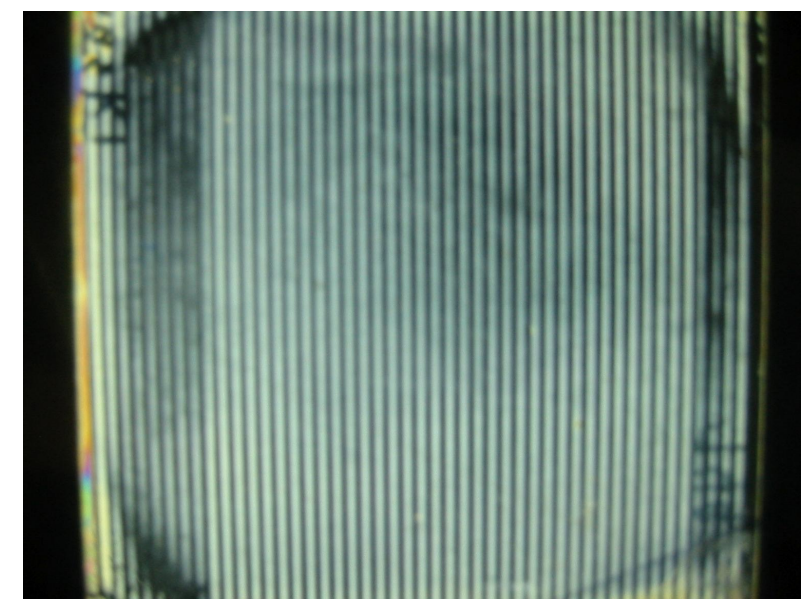

Fig.8. The thin film microretarder make by thermal \&UV lithography 
The gap between the mask and the film help to avoid the destruction of the film by the mask. However, it also applied the limits on the minimum achievable line width. As line width went smaller and smaller, the boundaries between the stripes become more and more vague.

In order to make microretarder with small-enough line width and clear boundary, we also tried laser scanning method. When Laser scanning method is adopted, a retarder plate with uniform retardation should be prepared in advance using the thermal UV process without mask. Then laser was used to remove the retardation of the unwanted area. Fig.9 is the result of laser scanning process. The black stripes are the area which retardation had been removed by laser scanning.

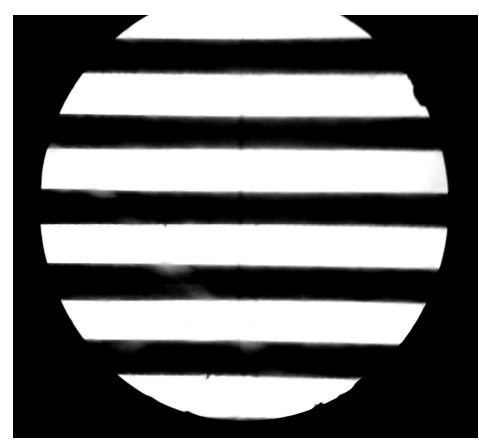

(a)

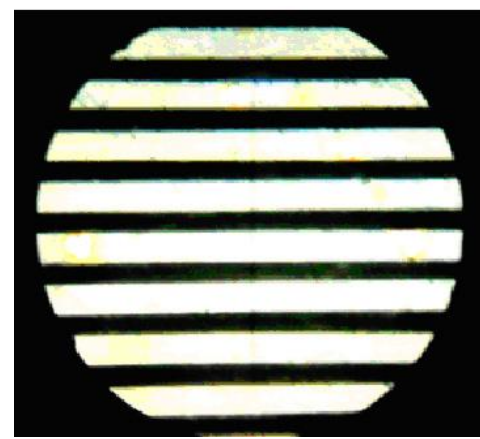

(b)

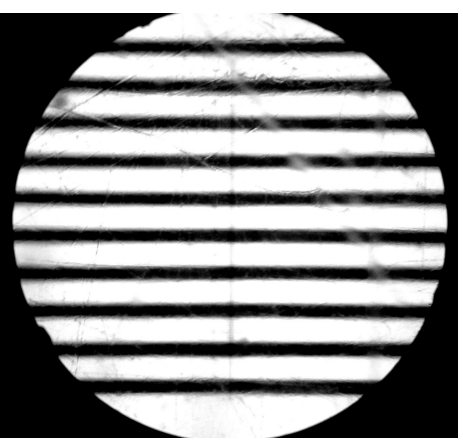

(c)

Fig.9 Photos of the laser scanned thin-film microretarder in microscope, with polarizer and analyzer (a) pitch $=300 \mu \mathrm{m},(\mathrm{b})$ pitch $=200 \mu \mathrm{m}$, (c)pitch $=150 \mu \mathrm{m}$

To verify the retardation distribution across the line width of the stripes, we use a focused laser beam to scan. He-Ne laser of $543 \mathrm{~nm}$ was used. The measured data were shown in Fig.10 . It was found that the transition area from high to low retardation is quite sharp. However, it is also found that there was still residual retardation in the scanned areas.

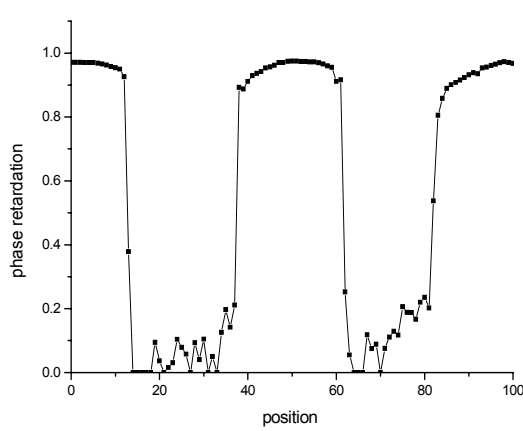

(a)

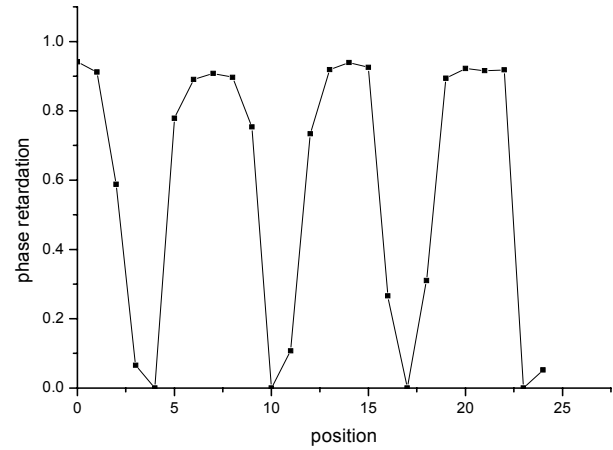

(b)

Fig. 10 the phase retardation distribution across the line width $\quad$.(a)pitch $=300 \mu \mathrm{m}$ (b)pitch=150 $\mu \mathrm{m}$

In this paper, we also worked on the experiments of verifying the compatibility of the retardation film of reactive liquid crystal with the processes of typical LC process. Table A and B are the retardation of the film before and after the 
process of alignment layer and thin film polarizer respectively.

\begin{tabular}{|c|c|c|}
\hline Alignment layer process & Before & After \\
\hline sample number & \multicolumn{2}{|c|}{ Phase retardation } \\
\hline 72003 & $0.986 \pi$ & $0.986 \pi$ \\
\hline 72007 & $0.986 \pi$ & $0.985 \pi$ \\
\hline
\end{tabular}

Table A. The phase retardation before and after the alignment layer process.

\begin{tabular}{|c|c|c|}
\hline Thin-film polarizer process & Before & After \\
\hline Sample number & \multicolumn{2}{|c|}{ Phase retardation } \\
\hline 72009 & $0.986 \pi$ & $0.856 \pi$ \\
\hline 72015 & $0.981 \pi$ & $0.866 \pi$ \\
\hline 72016 & $0.986 \pi$ & $0.855 \pi$ \\
\hline 72018 & $0.983 \pi$ & $0.847 \pi$ \\
\hline 72021 & $0.981 \pi$ & $0.845 \pi$ \\
\hline
\end{tabular}

Table B. The phase retardation changebefore and after the thin-film polarizer process.

As the results illustrated in Table A, the values of phase retardation didn't change after suffering the high temperature of the alignment layer process. However, in the procedure of the thin-film polarizer process shown in Table $B$, the retardation value changes a lot(from $0.98 \pi$ to $0.85 \pi$ roughly) because of the interaction of the material between the reactive liquid crystal and the thin-film polarizer.

\section{CONCLUSION AND FUTURE WORK}

In this paper, the process for fabricating retardation film of required retardation value using reactive liquid crystal material had been established. Two method of making micro-patterns on the retardation film were also tried by series of experiments. The compatibility of the film of reactive liquid crystal with the typical LC process was also tested on the processes of alignment layer and thin-film polarizer. It was found that laser scanning method is good in creating stripes pattern of small line width. Significant change of retardation value on the reactive liquid crystal layer was also found after the thin-film polarizer process. An isolation layer between the microretarder layer and the thin-film polarizer layer could solve the problem. In the next step, more compatibility evaluation experiments will be made in detail. When all the processes of making the retardation film into the in-cell structure are well established, an full-angle stereoscopic LCD will be easily implemented when the thin-film polarizer of high extinction ratio is available in the future.

\section{ACKNOWLEDGMENT}

Research on the microretarder for In-cell Stereoscopic LCD using Reactive Liquid Crystal technology has been supported by the Electronics and Opto-Electronics Lab of ITRI. The authors gratefully acknowledge the support of engineers in the EOL/ITRI..

\section{REFERENCES}

[1]Chao-Hsu Tsai, Kuen Lee, Kuo-Chung Huang, C.K. Lee, "Fabricating Polymeric Micro-retardation Arrays for Autostereoscopic Display System by CO2 Laser Heat Processing Technology"

[2] Wan-Jian Huang, Chao-Hsu Tsai, Ting-Ju Chen, Min-tsung Kuan, Chun-Hsiang Wen.” Development of the 
Random-Retardation-Encoding Anti-counterfeiting Technology" 